\section{Commentary: A potential strategy to improve lung graft function after ex vivo lung perfusion}

\author{
Roosevelt Bryant III, MD
}

In this issue of the Journal, Burki and colleagues ${ }^{1}$ from the University of Pittsburgh present the results of an elegant animal study assessing the impact of the drug triptolide on posttransplant outcomes in donor organs managed with ex vivo lung perfusion (EVLP). This study is particularly timely given the recent meta-analysis looking at the clinical impact of EVLP on the conversion rate of donor lungs for clinical lung transplantation. ${ }^{2}$ Twenty articles were included in the meta-analysis, accounting for 2574 donor organs and 2567 recipients. The salient findings were that the EVLP subgroup had a lower incidence of primary graft dysfunction $^{3}$ despite a longer intensive care unit stay. Other outcome measures, such as the need for posttransplant extracorporeal membrane oxygenation, ratio of $\mathrm{PaO}_{2}$ to inspired oxygen fraction, and 30-day survival, were not different between donor organs that were treated with EVLP and those that were not. ${ }^{2}$ The donor organ conversion rate to successful lung transplant with EVLP ranged between $34 \%$ and $100 \%$.

The question posed by the Pittsburgh group is this: Can the outcomes of EVLP be improved, so that it becomes more widely applicable ${ }^{3}$ Triptolide may provide a mechanism for improved EVLP outcomes. Burki and colleagues ${ }^{1}$ demonstrate, histologically and biochemically, that the use of triptolide in their EVLP model led to improved lung graft function. There was less alveolar edema histologically in the triptolide-treated lung transplants, lower levels of proinflammatory cytokines, and less oxidative stress. The clinical implication of these findings is a possible reduction in primary graft dysfunction, as indicated by an improved ratio

\footnotetext{
From the Division of Congenital Heart Surgery, The Heart Center, Phoenix Children's Hospital, Phoenix, Ariz.

Disclosures: Author has nothing to disclose with regard to commercial support.

Received for publication Jan 9, 2020; revisions received Jan 9, 2020; accepted for publication Jan 13, 2020; available ahead of print Jan 31, 2020.

Address for reprints: Roosevelt Bryant III, MD, Heart Transplantation and Mechanical Circulatory Support, Phoenix Children's Hospital, 1919 E Thomas Rd,

Phoenix, AZ 85016 (E-mail: rbryant1@phoenixchildrens.com).

J Thorac Cardiovasc Surg 2021;161:e75

$0022-5223 / \$ 36.00$

Copyright (c) 2020 by The American Association for Thoracic Surgery

https://doi.org/10.1016/j.jtcvs.2020.01.035
}

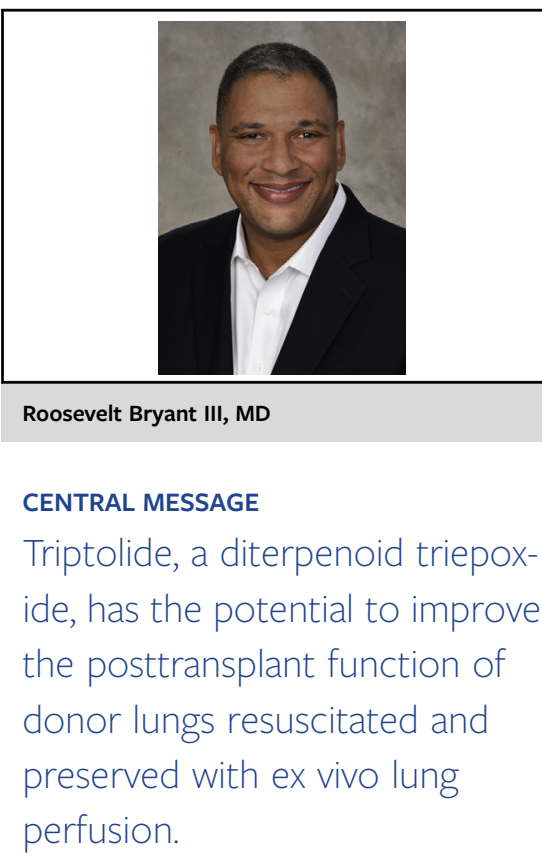

of $\mathrm{PaO}_{2}$ to inspired oxygen fraction. Given the emerging clinical evidence suggesting a benefit of EVLP in terms of reduced primary graft dysfunction, there appears to be justification for a broader clinical investigation of the effect of triptolide on ELVP as bridge to clinical lung transplant. There are obvious limitations to the Pittsburgh study, which requires translation of the findings to human trials. But there is also a broader question about what role EVLP should play in optimizing the lung transplant donor pool, given its potential $\operatorname{cost}^{3,4}$ and the availability of other strategies, such as lung protective donor protocols. ${ }^{3,5}$ Notwithstanding these issues, triptolide's effect on preserving lung graft function in the study of Burki and colleagues ${ }^{1}$ should be given serious consideration by the lung transplant community.

\section{References}

1. Burki S, Noda K, Philips BJ, Velayutham M, Shiva S, Sanchez PG, et al. Impact of triptolide during ex vivo lung perfusion on grafts after transplantation in a rat model. J Thorac Cardiovasc Surg. 2021;161:e65-74.

2. Luo Q, Zhu L, Wang Y, Wang L, Lv W, Hu J. The conversional efficacy of ex vivo lung perfusion and clinical outcomes in patients undergoing transplantation of donor lungs by ex vivo lung perfusion: a meta-analysis. Ann Transplant. 2019; 24:647-60.

3. Harrison MS, Frye CC, Puri V. Commentary: outcomes and transferability of ex vivo lung perfusion. J Thorac Cardiovasc Surg. 2020;159:356-7.

4. Fisher A, Andreasson A, Chrysos A, Lally J, Mamasoula C, Exley C, et al. An observational study of donor ex vivo lung perfusion in UK lung transplantation: DEVELOP-UK. Health Technol Assess. 2016;20:1-276.

5. Chang SH, Kreisel D, Marklin GF, Cook L, Hachem R, Kozower BD, et al Lung focused resuscitation at a specialized donor care facility improves lung procurement rates. Ann Thorac Surg. 2018;105:1531-6. 